\title{
The Canadian Shift: Still shifting?
}

\author{
Céleste Peterka \\ University of Ottawa
}

Previous literature on the Canadian Shift describes this phenomenon as a change in progress in many dialects of Canadian English. However, elements of the shift are not found to be consistent, particularly in the lowering of $[\mathrm{I}]$ and $[\varepsilon]$ and the retraction of [æ]. This paper investigates apparent time data from eight native speakers of Canadian English from the Ottawa area to investigate the nature of the shift in the region, as well as to better understand how the shift is manifested here compared to previous literature. Results presented in this paper, which were collected as part of an ongoing study, show that younger speakers produce only [I] and $[\varepsilon]$ vowels more retracted than older speakers. These data will later be compared to results of a perception study in order to investigate the relationship between perception and production of a sound change in progress.

\section{The nature of the Canadian Shift}

The Canadian Shift is an established phenomenon present in many dialects of Canadian English (SadlierBrown and Tamminga 2008). The Canadian Shift is described as a pull-chain involving the front-lax vowel system. The shift involves the lowering and retraction of [I] and [ $\varepsilon]$ (higher F1/lower F2), the retraction of [æ] (lower F2), and the centralization of $[\Lambda]$ (Clarke et al. 1995). This pull-chain is illustrated in Figure 1.

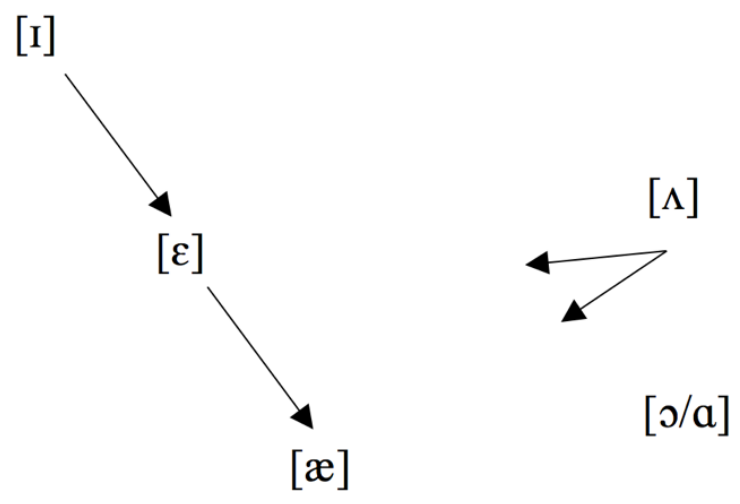

Figure 1. The Canadian Shift (Adapted from Clarke et al. 1995)

The cause of this vowel shift is possibly related to the low-back merger, in which the vowel [0] merges with [a] in many dialects of Canadian English. With these two back vowels merging, a space is created within the vowel system. According to the vowel dispersion principle, the space vowels occupy is evenly spread out (Ashby and Maidment 2005). When a change in the vowel system like the low-back merger occurs, the remaining vowels may shift to accommodate the opening (Sadlier-Brown and Tamminga 2008). 


\subsection{Previously on the Canadian Shift}

While further investigating what Labov (1991) described as the Northern Cities Vowel Shift, Clarke et al. (1995) find evidence that there is a similar shift occurring with Canadian English vowels, though the vowels are shifting in the opposite direction. ${ }^{1}$ This study enforces the apparent time hypothesis which is used to illustrate language change across generations by investigating speakers of different ages at a particular time. In this study, Clarke et al. (1995) investigate the Canadian Shift using impressionistic data from Ontarians, where they found evidence that younger speakers of Canadian English do produce shifted vowels as predicted by the Canadian Shift.

More recent studies use acoustic data and also find evidence of the Canadian Shift across Canada, for example, in Toronto and Thunder Bay (Roeder 2012), Montreal (Boberg 2005; Kettig and Winter 2017), as well as Vancouver and Halifax (Sadlier-Brown and Tamminga 2008).

In determining the geographical diffusion of the Canadian Shift in cities and rural communities, Roeder (2012) investigates speakers under 25 years old from Toronto and Thunder Bay to determine if the Canadian Shift is evident when comparing rural and urban locations. In terms of vowel production, there is very little difference between the two cities, though speakers from Thunder Bay had slightly less shifted $[\varepsilon]$ and $[æ]$ vowels than their counterparts in Toronto. This study also looks at Thunder Bay in apparent time, and the results from the shifting of younger speakers' vowels are not significant compared to older speakers, with the exception of the [a] vowel, where younger speakers have a significant retraction compared to older speakers.

Boberg (2005) seeks to improve upon the methodology from Clarke et al.'s (1995) study, as well as to expand on the data collected from Ontario by collecting data from Montreal. Using data from native English speakers from three generational groups, Boberg (2005) finds evidence that speaker age is a relevant factor in the production of the Canadian Shift where younger speakers produce more shifted vowels. In contrast to Clarke et al. (1995), however, this study found the most salient aspect of the shift to be the retraction of [I] and [ع], and not their lowering. A few years later, Kettig and Winter (2017) investigate the Canadian Shift in Montreal through apparent time to determine if, and how, it may have changed over the years since Boberg's (2005) study. This study found statistically significant results for the lowering and the retraction of $[\varepsilon]$, as well as the retraction of [æ]. While the results are slightly different than earlier studies from Montreal, they do suggest that the Canadian Shift is still active in some ways in more recent years.

To determine the effect of the Shift across Canada, Sadlier-Brown and Tamminga (2008) investigate the Canadian Shift in Vancouver and Halifax. Apparent time data are collected from 26 speakers and provide evidence of the shift on both the East and West coasts of Canada. In addition to the retraction of [I] and $[\varepsilon]$, this study also finds the vowels are produced significantly lower by younger speakers, which is similar to findings from Clarke et al. (1995).

These studies provide evidence that the Canadian Shift may not be active in the way it is described by Clarke et al. (1995), and may differ in terms of urban and rural areas. As Ottawa is a city which has urban, suburban, and rural areas, investigating the linguistic characteristics of the Canadian Shift here can provide more insight on the nature of the shift as a whole. Additionally, while previous literature suggests the Canadian Shift to still be active across Canada, acoustic evidence from these speakers suggests it may be transitioning from a change in progress to a completed sound change.

\section{The current experiment}

The current experiment investigates the nature of the Canadian Shift as spoken by native speakers of Canadian English from Ottawa, Ontario. Ottawa provides a unique geographical and linguistic setting for which investigating conflicting elements of the Canadian Shift may prove useful. Particularly, due to its layout, the city includes urban, suburban, and rural areas. Previous literature has shown confounding results

\footnotetext{
${ }^{1}$ The Northern Cities Vowel Shift is not exactly the opposite of the Canadian Shift, as it seems to be only affecting low vowels $[\mathfrak{x}]$ and $[\mathrm{a} / \mathrm{o}]$.
} 
when investigating urban and rural communities, where age-related differences in vowel production are not statistically significant (e.g., Roeder 2012). Because of this, it may be useful to investigate one city with such a variety of communities in effort to tease apart the effect of age and the Canadian Shift. To do so, this study seeks to answer the following research questions:

(1) a. In Ottawa, do younger Canadians produce more shifted vowels than older Canadians?

b. Are there age-related differences in how vowels in the Canadian Shift are produced in Ottawa?

In order to address these questions, a production experiment was conducted on eight participants, four of which were between 18-30 years old, and four which were over the age of 60 .

\subsection{Methodology}

\subsubsection{Participants}

The current experiment investigated eight native, monolingual speakers of Canadian English from Ottawa. Four were between the ages 18-30, and four were over 60 years old. ${ }^{2}$ All participants were recruited through social media (reddit, Facebook, and Instagram), or through word of mouth. Information collected from each participant includes their age and where they grew up.

\subsubsection{Stimuli}

The stimuli included the entirety of the Canadian English monophthong vowel system ([i, I, $\varepsilon, \mathfrak{x}, \mathrm{u}, \mho, \Lambda$, a]) situated in monosyllabic words between combinations of voiced and voiceless bilabial, alveolar, and velar stops. The carrier word occurred sentence-final, and there were a total of 103 sentences. While all monophthongs were included, this was just to provide a more complete picture of the vowel space, which is relevant when normalizing formant values from multiple speakers, and the results show only the vowels

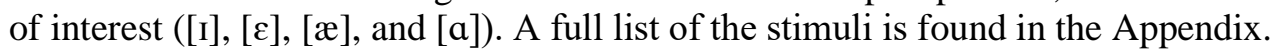

Recordings were conducted in the Sound Patterns Laboratory's sound-attenuated booth at the University of Ottawa using a Philips Omnidirectional Handheld microphone (LBB 9080/55 NR552) and recorded in Audacity® (Dannenberg and Mazzoni 2015). The stimuli were presented through PowerPoint, each sentence was shown on a slide and the slides were advanced at the participant's own pace, the sentences were read through three times, and were randomized each time.

\subsection{Acoustic analysis}

Acoustic analyses for the data were conducted in Praat [V. 6.0.43] (Boersma and Weenink 2018). After importing the sound files, text grids were created manually for each of the sentence-final target words. The formant values (F1, F2, and F3) at the beginning, midpoint, and at the end of the vowels were automatically extracted using a script (Buss 2014). The results and analyses presented below focus only on the midpoint formant values.

\footnotetext{
${ }^{2}$ The results presented in this paper were collected as part of a larger experiment investigating the interface between perception and production. The final study will include 10 participants in each of the two age categories who complete both a production and a perception task relating to the Canadian Shift.
} 


\section{Results}

\subsection{Establishing the vowel space}

The raw formant values from each token and speaker were normalized using the Nearey 1 method (Thomas and Kendall 2007) to look for any initial trends amongst the speakers. ${ }^{3}$ An example word was chosen to represent each of the vowel sounds in the plot below, such that beet represents [i], bit for [I], bet for [E], bat

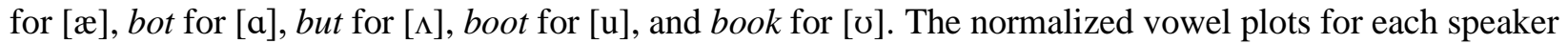
group are shown in Figure 2, younger speakers are shown in grey while older speakers are in black.

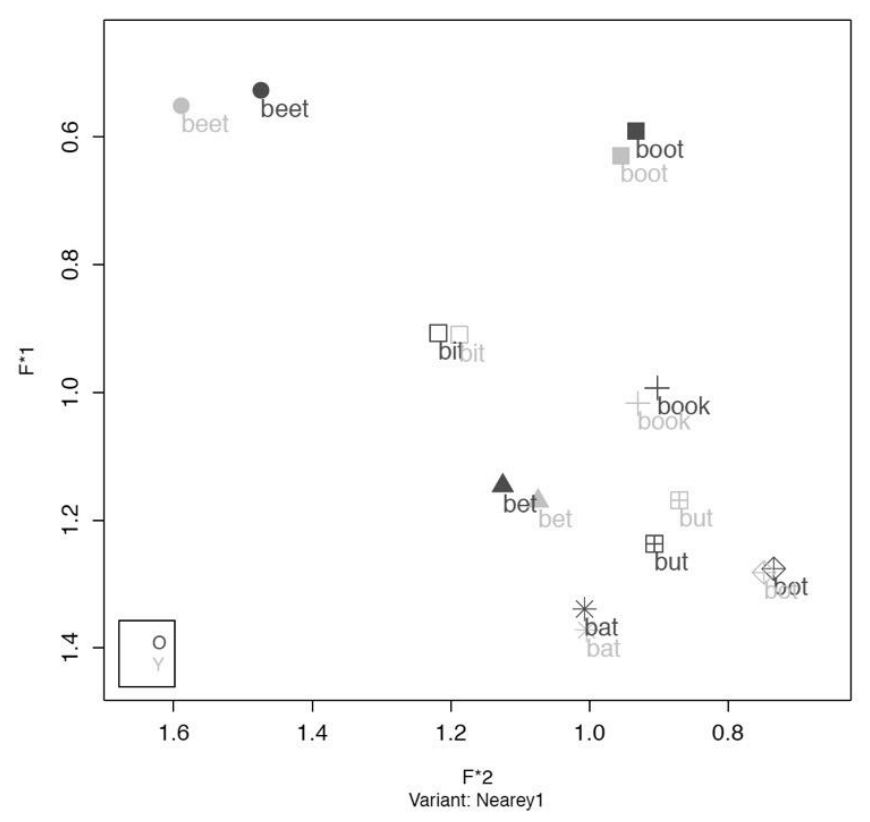

Figure 2. Normalized F1 and F2 values for younger and older speaker groups for all vowels

It is important to discuss the logistics of using the Nearey 1 normalization formula. An advantage for Nearey 1 is that it provided normalized formant values that filter out physiological differences and maintain sociolinguistic variation. However, there are also disadvantages. These include the possibility of a skewed plot if an incomplete vowel inventory is provided, and individual dialectal differences can also result in skewedness. As this plot shows the values from just eight speakers, who did not produce diphthongs, the plot can be skewed. However, the plot is a useful tool to illustrate the comparison of two group's vowel space, for initial observations. Based on this plot, the younger speaker group's bit vowel does not appear much lower, but it is slightly more back. The bet vowel is slightly lower and more retracted, the bat vowel is lower, and the bot vowel is nearly overlapping between the two groups. These observations are further investigated through statistical analysis.

\subsection{Mixed effects linear regression results}

The normalized values for the vowels of interest ([I], $[\varepsilon],[æ]$, and $[\mathrm{a}])$ were used to run several mixed effects linear regression in R [V 3.5.1] (R Core Team 2018). In each model, the dependent variable was

${ }^{3}$ The Nearey 1 method for normalization is formant intrinsic, and uses the formula $\mathrm{F}_{n[V]}{ }_{n[V}=\operatorname{anti}-\log \left(\log \left(\mathrm{F}_{n[V]}\right)-\right.$ mean $\left.\left(\log \left(F_{n}\right)\right)\right)$. 
either the normalized F1 or F2 value, and the independent variables were age group (young) and speaker mean F0 (not reported here).

The statistical hypotheses in (3) and (4) were used to determine how to appropriately answer the research questions from (1), which are restated in (2).

(2) Broad Research Questions

a. In Ottawa, do younger Canadians produce more shifted vowels than older Canadians?

b. Are there age-related differences in how vowels in the Canadian Shift are produced in Ottawa?

(3) Null Hypothesis

$\mathrm{H}_{0}$ No statistically significant difference in formant values (F1 or F2) between younger and older speakers.

(4) Alternative Hypotheses

a. $\mathrm{H}_{1}$ Younger speakers produce $[\mathrm{I}]$ and $[\varepsilon]$ lower and more retracted (higher F1, lower F2).

b. $\mathrm{H}_{1}$ Younger speakers produce [æ] more retracted (lower F2).

c. $\mathrm{H}_{1}$ Younger speakers produce [a] lower and more retracted (higher F1 and lower F2).

\subsubsection{The statistical models}

Each vowel was run in its own model, with separate models for F1 and F2. ${ }^{4}$ The following eight tables show each vowel's mixed effects linear regression models for F1 and F2. For these models, the older age group serves as a baseline. These results are discussed further in Sections 3.2.2-3.2.4. ${ }^{5}$

Table 1. Statistical model for $[\mathrm{I}]$ and $\mathrm{F} 1(\mathrm{df}=357)$

\begin{tabular}{ccccc} 
Coefficients & Estimate & Std. Error & $t$-value & $p$-value \\
\hline Intercept & 0.9731 & 0.0574 & 16.937 & $<0.0001$ \\
\hline agegroupyoung & 0.0127 & 0.0237 & 0.537 & 0.5916 \\
\hline meanF0 & -0.0004 & 0.0003 & -1.354 & 0.1765 \\
\hline
\end{tabular}

Table 2. Statistical model for $[\mathrm{I}]$ and $\mathrm{F} 2(\mathrm{df}=357)$

\begin{tabular}{ccccc} 
Coefficients & Estimate & Std. Error & $t$-value & $p$-value \\
\hline Intercept & 1.0316 & 0.0471 & 21.879 & $<0.0001$ \\
\hline agegroupyoung & -0.0441 & 0.0196 & -2.246 & 0.0253 \\
\hline meanF0 & 0.0012 & 0.0003 & 4.213 & $<0.0001$ \\
\hline
\end{tabular}

\footnotetext{
${ }^{4}$ An example of the model for bit vowel is > model.bit.F1.b <- $\operatorname{lmer}($ nenormF1 $\sim$ agegroup + meanF0 $+(1 \mid$ speaker) $+(1 \mid$ actual.word), data=data.bit, control=lmerControl(optimizer="bobyqa", optCtrl=list(maxfun=2e5))).

5 The p-values were calculated using a t-value to p-value online calculator.
} 


\section{CÉLeste Peterka}

Table 3. Statistical model for $[\varepsilon]$ and F1 $(\mathrm{df}=257)$

\begin{tabular}{ccccc} 
Coefficients & Estimate & Std. Error & $t$-value & $p$-value \\
\hline Intercept & 1.0258 & 0.0811 & 12.638 & $<0.0001$ \\
\hline agegroupyoung & 0.0076 & 0.3379 & 0.226 & 0.8213 \\
\hline meanF0 & 0.0007 & 0.0004 & 1.582 & 0.1148 \\
\hline
\end{tabular}

Table 4. Statistical model for $[\varepsilon]$ and F2 $(\mathrm{df}=257)$

\begin{tabular}{ccccc} 
Coefficients & Estimate & Std. Error & $t$-value & $p$-value \\
\hline Intercept & 1.0051 & 0.0523 & 19.188 & $<0.0001$ \\
\hline agegroupyoung & -0.0615 & 0.0211 & -2.904 & 0.004 \\
\hline meanF0 & 0.0007 & 0.0003 & 2.523 & 0.0122 \\
\hline
\end{tabular}

Table 5. Statistical model for [æ] and F1 $(\mathrm{df}=400)$

\begin{tabular}{ccccc} 
Coefficients & Estimate & Std. Error & $t$-value & $p$-value \\
\hline Intercept & 1.1375 & 0.1177 & 9.658 & $<0.0001$ \\
\hline agegroupyoung & 0.0057 & 0.0497 & 0.116 & 0.9077 \\
\hline meanF0 & 0.0013 & 0.0007 & 1.793 & 0.0737 \\
\hline
\end{tabular}

Table 6. Statistical model for $[æ]$ and F2 $(\mathrm{df}=400)$

\begin{tabular}{ccccc} 
Coefficients & Estimate & Std. Error & $t$-value & $p$-value \\
\hline Intercept & 1.0000 & 0.0065 & 15.313 & $<0.0001$ \\
\hline agegroupyoung & -0.0004 & 0.0027 & -0.147 & 0.8832 \\
\hline meanF0 & $<0.0001$ & $<0.0001$ & 0.107 & 0.9148 \\
\hline
\end{tabular}

Table 7. Statistical model for $[\mathrm{a}]$ and $\mathrm{F} 1(\mathrm{df}=381)$

\begin{tabular}{ccccc} 
Coefficients & Estimate & Std. Error & $t$-value & $p$-value \\
\hline Intercept & 1.4587 & 0.0725 & 20.12 & $<0.0001$ \\
\hline agegroupyoung & 0.0192 & 0.0311 & 0.619 & 0.5362 \\
\hline meanF0 & -0.0012 & 0.0004 & -2.653 & 0.0083 \\
\hline
\end{tabular}


THE CANADIAN SHIFT: STILL SHIFTING?

Table 8. Statistical model for $[\mathrm{a}]$ and $\mathrm{F} 2(\mathrm{df}=381)$

\begin{tabular}{ccccc} 
Coefficients & Estimate & Std. Error & $t$-value & $p$-value \\
\hline Intercept & 0.8403 & 0.0902 & 9.313 & $<0.0001$ \\
\hline agegroupyoung & 0.0226 & 0.0388 & 0.581 & 0.5615 \\
\hline meanF0 & -0.0007 & 0.0005 & -1.237 & 0.2168 \\
\hline
\end{tabular}

\subsubsection{Age}

Speaker age is of interest in accordance to previous literature on the Canadian Shift as younger speakers are shown to produce more shifted vowels than older speakers (e.g., Boberg 2005; Sadlier-Brown and Tamminga 2008; Roeder 2012; Kettig and Winter 2017). If the Canadian Shift is active in Ottawa in a similar way to previous literature, we could expect the lowering and retracting of $[\mathrm{I}]$ and $[\varepsilon]$, and the retracting of $[\mathfrak{e}]$ and $[a]$.

\subsubsection{Age and F1}

In terms of lowering (higher F1), no vowel is significantly lower, as illustrated above in Tables 1, 3, 5, and 7. This result is expected based on Boberg (2005) and Roeder (2012), while it differs from Sadlier-Brown and Tamminga's (2008), as well as Kettig and Winter's (2017) findings where $[\mathrm{I}]$ and $[\varepsilon]$ do lower significantly in younger speakers.

\subsubsection{Age and F2}

In terms of retraction (lower F2), the results of the linear regression suggest significant retraction of [I] and [ع], shown above in Tables 2 and 4, though no significant retraction for [æ] or [a], as illustrated in Tables 6 and 8. The retraction of [I] and [ع] is expected based on previous literature, while the behaviour of [æ] and $[a]$ is more unexpected. Based on previous literature, we could expect [æ] and [a] to be more retracted in what De Decker (2010) describes as the [æ]-backing chain shift, which is also found to be significant in Kettig and Winter (2017). However, this is not evident based on the data provided by these eight speakers.

\section{Discussion}

For the vowels involved in the Canadian Shift that were included in this analysis ([I], [ع], [æ], and [a]), we would expect older speakers to produce [I] and [ $[\varepsilon]$ more fronted, and possibly higher, [æ] would be produced more fronted, and [a] would be produced higher than younger speakers (Clarke et al. 1995; Boberg 2005; Sadlier-Brown and Tamminga 2008; Roeder 2012; Kettig and Winter 2017).

The eight speakers' data reported here provide insight on the Canadian Shift in Ottawa. It seems to take on a similar flavour as earlier studies from Montreal (Boberg 2005), and from Toronto and Thunder Bay (Roeder 2012), though different from very early work from Clarke et al. (1995), as well as more recent work (e.g., Sadlier-Brown and Tamminga 2008; Kettig and Winter 2017).

In this experiment, in terms of lowering, the younger speaker group did not produce any statistically lower vowels than the older speakers. Retraction was only significant for [I] and [ $\varepsilon]$, and the retraction of the other vowels was not statistically significant.

In terms of the statistical hypotheses in (3) and (4), the null hypothesis, that there is no significant difference in formant values (F1 or F2) between younger and older speakers, cannot be rejected for [æ] or [a] as there was no significant difference for F1 or F2 in younger speakers. However, in terms of [I] and [ع] 
the null hypothesis can be rejected as there was a significant difference in one of the formant values (F2) as produced by younger speakers.

As for the research questions outlined in (1), these can be tentatively answered given the results from the current sample population. In general, younger Canadians do produce more shifted vowels than older Canadians as expected based on previous literature. While significance was not found except for the retraction of [I] and $[\varepsilon]$, it is possible that this result can change with further analysis of the other 12 speakers in this study. For the second research question, there is evidence that age is a significant factor in predicting shifted vowels in accordance to what is expected with the Canadian Shift. At this point, these data suggest the Canadian Shift is active to some extent in Ottawa.

\subsection{Next steps and future research}

As this is an ongoing experiment, further data analysis is required before determining the behaviour of the Canadian Shift in Ottawa. Incorporating the remaining data from 12 other speakers will provide a better understanding of the way the Canadian Shift is produced in Ottawa. Specifically, more data may illustrate how younger speakers are producing the Shift, to what extent its behaviour is expected based on previous literature, as well as how to determine if the Shift is still an ongoing change in progress.

The data presented here show only a piece of what the Canadian Shift looks like in Ottawa. Not only due to a small sample size, but also in regards to the other side of a change in progress - perception. Each of the participants in this experiment also completed a perception task, and final versions of this study will include the relationship between perception and production on a group and individual level.

\section{Conclusion}

What appears most salient from these data was how younger speakers produced a significant retraction of [I] and [E]. The other vowels involved in the Canadian Shift were not significantly shifted when produced by younger speakers, which at this point, only conflates the issue of inconsistencies from previous research in production. It is possible that Ottawa's mixture of urban and rural identities contributes to the manifestation of the Shift, as it did in Roeder's (2012) study. However, further analysis is required in order to determine the status of the Canadian Shift in Ottawa. If any conclusions can be made at this point, it is that there is still much to investigate on the Canadian Shift, and the way it is understood now may not be fully representative of what exactly this phenomenon entails.

Acknowledgements. I would like to express my gratitude and appreciation to my supervisor, Dr. Kevin McMullin, who helped in every step of this experiment, but particularly in refining the methodology of this experiment and for being extremely patient while I try and learn how to use R. I would also like to thank Dr. Marc Brunelle who reviewed the pilot version of this experiment and provided helpful comments and suggestions. I'd also like to thank all my participants for leaving their vowels in my hands, as well as everyone at MOT 2019 at the University of Toronto for accepting this presentation for the Phonetics and Phonology Workshop and providing a supportive environment for helpful feedback.

\section{References}

Ashby, Michael, and John Maidment. 2005. Introducing phonetic science. Cambridge: Cambridge University Press.

Boberg, Charles. 2005. The Canadian shift in Montreal. Language Variation and Change 17(2): 133-154.

Boersma, Paul, and David Weenink. 2018. Praat: Doing phonetics by computer (Version 6.0.43) [Computer software]. http://praat.org. 
Buss, Steven. 2014. Sbuss/get-formants-for-vowels. Retrieved from https://github.com/sbuss/get-formantsfor-vowels.

Clarke, Sandra, Ford Elms, and Amani Youssef. 1995. The third dialect of English: Some Canadian evidence. Language Variation and Change 7(2): 209-228.

De Decker, Paul. 2010. Sounds shifty: Gender and age differences in perceptual categorization during a phonetic change in progress. University of Pennsylvania Working Papers in Linguistics 15(2): 48-60.

Feinberg, David. R. 2018. Measure pitch, jitter, shimmer, and HNR. Retrieved from https://osf.io/dbrpf/wiki/home/.

Kettig, Thomas, and Bodo Winter. 2017. Producing and perceiving the Canadian Vowel Shift: Evidence from a Montreal Community. Language Variation and Change 29(1): 79-100.

Mazzoni, Dominic, and Roger Dannenberg. 2015. Audacity (Version 2.1.0) [Computer software]. https://www.audacityteam.org.

Ottawa Rural Communities. 2002. Retrieved from http://www.ruralcouncil.ca/ORC/background.htm.

P Value from T-Score Calculator. 2019. Retrieved from https://www.socscistatistics.com/pvalues/tdistribution.aspx.

R Core Team. 2018. R: A language and environment for statistical computing. R Foundation for Statistical Computing, Vienna, Austria. https://www.R-project.org/.

Roeder, Rebecca. 2012. The Canadian Shift in two Ontario cities. World Englishes 31(4): 478-494.

Sadlier-Brown, Emily, and Meredith Tamminga. 2008. The Canadian Shift: Coast to coast. Proceedings of the 2008 Annual Conference of the Canadian Linguistic Association, 1-14.

Thomas, Erik. R, and Tyler Kendall. 2007. NORM: The Vowel Normalization and Plotting Suite. http://lingtools.uoregon.edu/norm/index.php. 


\section{Appendix: Stimuli (Sentences)}

[i]

[I]

[ع]

[æ]

1. This is Pete.

1. The cherry has a pit.

1. The dog was pet.

1. This is Pat.

2. Don't make a peep.

2. Take your pick.

2. They're full of pep.

2. I bought a lunch pack.

3 . They reached the

3. I left a tip.

3. I'm going to bed.

3. Turn off the tap.

peak.

4. Watch for ticks.

4. Dogs always beg.

4. It fell off the tack.

4. That's the goalie's

5. I have a kit.

5. The plant is dead.

5. I wore a cap.

keep.

6 . The horse didn't

6. I found the peg.

6. I love my cat.

kick.

7. This is Ted.

7. That would be bad.

6 . The cat peed.

7. The baby wears a

8. I lost the bet.

8. I lost my bag.

7. The car was keyed.

bib.

8 . The duck has a beak.

9. I paid off my debt.

9. I called my dad.

9. I grow beets.

8. I would like to bid.

10. I washed the deck.

10. The smell makes me

9. The dog is big.

11. This math I don't

gag.

10. I don't know what I

get.

11. I have a pad.

did.

12. Put it on my tab.

11. I will start to dig.

12. The band got a gig.

13. I lost the tag.

14. I took a cab.

13. They have one kid.

15 . Watch your back.

14. I think you were bit.

16. I'm up to bat.

15. I want to take a dip.

17. I jumped the gap.

[a]

[u]

[v]

$[\Lambda]$

1. The pasta is in the pot.

2. I am drinking pop.

1. Watch out for dog

poop.

1. There's something I

he puck.

3. Some skills must be

2. The horn sounds like a took.

taught.

toot.

2. They're the cook.

2. This dog is just a

4. The answer is at the

top.

3. I bought a chicken

3 . It turned out to be

pup.

good.

coop.

5. A baby is still learning

to talk.

4. The crowd booed.

5. You're a good dude.

4. I did all I could.

5. I love this book.

6. The woman is a cop.

7. This is the fish I

6. I have a long tube.

caught.

7. The doves cooed.

8. I lost a boot.

8 . The river turns into a

bog.

9. The pudding turned into goop.

9. I would like to have a

dog.

10. This is Todd.

11. I like corn on the cob.

12. I showed you what I

bought.

13. I caught a cod.

14. This is a blue dot.

15. I'll meet you on the

dock.

16. Show me what you

got.

3 . This is your cup.

4. The paper was

cut.

5. You're my best bud.

6 . I caught a bug.

7. This battery is a dud.

8. Look at this hole I

dug.

9. Meet me at the

pub.

10. I have a pug.

11. Water filled the

tub.

12. Give it a tug.

13. A baby bear is a

cub.

14. Cows lick the

cud.

15. It costs a buck.

16. This is my duck.

17. Always trust

your gut. 